This item was submitted to Loughborough's Research Repository by the author.

Items in Figshare are protected by copyright, with all rights reserved, unless otherwise indicated.

\title{
From quantum disorder to magnetic order in an $s=1 / 2$ kagome lattice: a structural and magnetic study of herbertsmithite at high pressure
}

PLEASE CITE THE PUBLISHED VERSION

http://dx.doi.org/10.1103/PhysRevLett.108.187207

\section{PUBLISHER \\ (C) American Physical Society}

VERSION

VoR (Version of Record)

\section{PUBLISHER STATEMENT}

This work is made available according to the conditions of the Creative Commons Attribution-NonCommercialNoDerivatives 4.0 International (CC BY-NC-ND 4.0) licence. Full details of this licence are available at: https://creativecommons.org/licenses/by-nc-nd/4.0/

\section{LICENCE}

CC BY-NC-ND 4.0

\section{REPOSITORY RECORD}

Kozlenko, D.P., Anna F. Kusmartseva, E.V. Lukin, D.A. Keen, W.G. Marshall, M.A. DeVries, and Konstantin V. Kamenev. 2019. "From Quantum Disorder to Magnetic Order in an $S=1 / 2$ Kagome Lattice: A Structural and Magnetic Study of Herbertsmithite at High Pressure". figshare. https://hdl.handle.net/2134/17366. 


\title{
From Quantum Disorder to Magnetic Order in an $s=1 / 2$ Kagome Lattice: A Structural and Magnetic Study of Herbertsmithite at High Pressure
}

\author{
D. P. Kozlenko, ${ }^{1}$ A. F. Kusmartseva, ${ }^{2}$ E. V. Lukin, ${ }^{1}$ D. A. Keen, ${ }^{3}$ W. G. Marshall, ${ }^{3}$ M. A. de Vries, ${ }^{2}$ and K. V. Kamenev ${ }^{4}$ \\ ${ }^{1}$ Frank Laboratory of Neutron Physics, Joint Institute for Nuclear Research, 141980 Dubna, Russia \\ ${ }^{2}$ CSEC and School of Chemistry, The University of Edinburgh, Edinburgh, EH9 3JZ, United Kingdom \\ ${ }^{3}$ ISIS Facility, STFC Rutherford Appleton Laboratory, Harwell Oxford, Oxon, OX11 OQX, United Kingdom \\ ${ }^{4}$ CSEC and School of Engineering, The University of Edinburgh, Edinburgh, EH9 3JZ, United Kingdom
}

(Received 26 October 2011; published 3 May 2012)

\begin{abstract}
The structural and magnetic properties of deuterated herbertsmithite have been studied by means of neutron powder diffraction and magnetic susceptibility measurements in a wide range of temperatures and pressures. The experimental data demonstrate that a phase transition from the quantum-disordered spinliquid phase to the long-range ordered antiferromagnetic phase with the Néel temperature $T_{N}=6 \mathrm{~K}$ is induced at $P=2.5 \mathrm{GPa}$. The observed decrease of $T_{N}$ upon compression correlates with the anomalies in pressure behavior of $\mathrm{Cu}-\mathrm{O}$ bond length and $\mathrm{Cu}-\mathrm{O}-\mathrm{Cu}$ bond angles. The reasons for the observed spinfreezing transition are discussed within the framework of the available theoretical models and the recent observation of the field-induced spin freezing.
\end{abstract}

DOI: 10.1103/PhysRevLett.108.187207

PACS numbers: $75.25 .-\mathrm{j}, 61.50 . \mathrm{Ks}, 75.40 . \mathrm{Cx}$

The spin-1/2 corner-sharing kagome antiferromagnetic lattice has become a frontier in the search for novel quantum phenomena in condensed matter over the past two decades [1]. One of the most interesting effects observed in this system is the realization of the quantum spin-liquid state, in which the classical Néel long-range magnetic order is destabilized by quantum fluctuations $[1,2]$.

Among the model systems with the spin- $1 / 2$ kagome lattice, the mineral herbertsmithite $\mathrm{ZnCu}_{3}(\mathrm{OH})_{6} \mathrm{Cl}_{2}$ has recently been highlighted as the most "structurally perfect" realization [3]. Its structure contains $\mathrm{Cu}^{2+}$-based kagome planes, separated by nonmagnetic $\mathrm{Zn}^{2+}$ ions. This is the only kagome model system with the highest value of frustration index $(f>157)$, which does not exhibit any (partial) freezing of the spins down to at least $50 \mathrm{mK}$ [4] despite significant nearest-neighbor exchange estimated at $J=$ 190 K [3-6]. Neutron scattering experiments revealed the presence of instantaneous short-ranged antiferromagnetic (AFM) correlations in the absence of a time-averaged ordered magnetic moment - the key feature of the quantum spin liquid [7]. It has also been shown experimentally that kagome layers in herbertsmithite have a nonzero susceptibility at $T \rightarrow 0 \mathrm{~K}[8,9]$, which is in agreement with the continuum of quantum excitations observed using neutron spectroscopy [5,7]. Among the important factors potentially affecting the properties of herbertsmithite are the Dzyaloshinsky-Moriya interaction [10] and a small degree of antisite disorder, involving mixing of $\mathrm{Cu}^{2+}$ and $\mathrm{Zn}^{2+}$ ions between $\mathrm{Cu}$ and $\mathrm{Zn}$ sites [11].

The continuous magnetic excitation spectrum [5,7-9] and the absence of magnetic order [4] have been attributed to the realization of or proximity to a quantum-critical phase or a quantum-critical point $[5,7]$. Recent theoretical calculations [12] predict a quantum phase transition to a spin-frozen state, induced by the Dzyaloshinsky-Moriya (DM) spin-spin interaction. This antisymmetric exchange interaction is allowed in herbertsmithite due to the absence of the inversion symmetry across the $\mathrm{Cu}-\mathrm{O}-\mathrm{Cu}$ superexchange pathways. The DM interaction might explain the field-induced spin freezing with $\mu_{0} H_{c} \sim 1.6 \mathrm{~T}$ observed using ${ }^{17} \mathrm{O}$ NMR. [13]. Strength of the DM interaction with respect to the magnetic superexchange depends strongly on the geometry, i.e., on the interatomic bonds and angles, of the $\mathrm{Cu}-\mathrm{O}-\mathrm{Cu}$ superexchange pathway. It might therefore be possible to bring about a DM interaction-induced spinfreezing transition by the application of hydrostatic pressure, which modifies the geometry of the superexchange interaction pathway. The aim of the experiments described in this Letter was to search for spin freezing in the spin-liquid material herbertsmithite under pressure. The pressure-induced spin-frozen states were previously observed in the 3D spin-liquid pyrochlore $\mathrm{Tb}_{2} \mathrm{Ti}_{2} \mathrm{O}_{7}$ [14], and also 1D spin-liquids $\mathrm{TlCuCl}_{3}$ [15] and $\mathrm{KCuCl}_{3}$ [16].

Deuterated herbertsmithite was synthesized by the hydrothermal method as described in Ref. [3]. The deuteration level of $97 \%$ and the level of $\mathrm{Cu} / \mathrm{Zn}$ antisite disorder, involving $6 \%$ of the $\mathrm{Cu}^{2+}$ ions and $18 \%$ of the $\mathrm{Zn}^{2+}$ ions, were estimated from neutron diffraction measurements performed at the GEM diffractometer (ISIS pulsed neutron source, Rutherford Appleton Laboratory, UK).

The crystal structure of herbertsmithite in the pressure range 0-5.1 GPa at ambient temperature was studied using the Pearl/HiPr diffractometer (ISIS, RAL, UK) and the Paris-Edinburgh pressure cell [17]. A TiZr encapsulated gasket of $75 \mathrm{~mm}^{3}$ initial volume and the $4: 1$ volume mixture of fully deuterated methanol-ethanol as a pressure-transmitting medium were used to attain nearly hydrostatic compression of the sample [18]. A small 
$\sim 1 \mathrm{~mm}$ diameter) pellet of polycrystalline lead was added to the sample to act as a suitable pressure marker [19]. Diffraction patterns were collected using the main PEARL/HiPr detector bank covering the angular range $83^{\circ}<2 \theta<97^{\circ}$. The data collection times were about 3 hours at each pressure.

The magnetic structure of herbertsmithite in the pressure range $0-3 \mathrm{GPa}$ and temperature range $1.4-300 \mathrm{~K}$ was studied using the GEM diffractometer (ISIS, RAL, UK). A sapphire-anvil high-pressure cell [20] with a sample volume of about $2 \mathrm{~mm}^{3}$ was used. Several tiny ruby chips were placed at different points of the sample surface to monitor the pressure distribution. The pressure was determined by the ruby fluorescence technique with the accuracy of $0.05 \mathrm{GPa}$ at each ruby chip and the pressure value on the sample was determined by averaging pressure values read off from the ruby chips. The estimated inhomogeneity of the pressure distribution on the sample surface was less than $15 \%$. Diffraction patterns were collected from a GEM detector bank located at an average scattering angle of $2 \theta=34.96^{\circ}$ and the typical data collection time was about 20 hours. The diffraction data were analyzed by the Rietveld method using the FULLPROF program [21].

The magnetic susceptibility measurements were performed with a miniature high-pressure cell for a SQUID magnetometer using a dc field of 100 Oe [22]. Bevelled diamonds with culets of $800 \mu \mathrm{m}$ were used as the anvils and a $250 \mu \mathrm{m}$ thick beryllium-copper foil indented to $100 \mu \mathrm{m}$ was used as the gasket. A hole of $350 \mu \mathrm{m}$ diameter was drilled in the gasket and filled with the sample. Daphne 7373 oil was used as the pressure-transmitting medium.

Additional electrical resistivity measurements were conducted by a four-probe technique in a small diamond anvil cell used with the Physical Property Measurement System (Quantum Design, USA) [23]. Diamonds with $200 \mu \mathrm{m}$ culets were used as the anvils and the probes were made of gold foil. The measurements were done in the range of temperatures of 2-300 K and at pressures of up to $35 \mathrm{GPa}$.

Neutron diffraction patterns of deuterated herbertsmithite obtained at selected pressures and ambient temperature on the Pearl/HiPr diffractometer and refined by the Rietveld method are shown in Fig. 1. The rhombohedral crystal structure of $R \overline{3} m$ symmetry remains stable in the investigated pressure range up to $5 \mathrm{GPa}$. The values of structural parameters obtained at ambient conditions agree well with previous studies $[3,11]$. The lattice compression is anisotropic with the linear compressibilities $k_{a i}=$ $-\left(1 /\left(a_{i}\right)_{P=0}\right)\left(d a_{i} / d P\right)_{T}$ of lattice parameters $k_{a}=$ 0.0050 and $k_{c}=0.0026 \mathrm{GPa}^{-1}$. The unit cell volume as a function of pressure was fitted by the Birch-Murnaghan equation of state [24]

$P=\frac{3}{2} B_{0}\left(x^{-(7 / 3)}-x^{-(5 / 3)}\right)\left[1+\frac{3}{4}\left(B^{\prime}-4\right)\left(x^{-(2 / 3)}-1\right)\right]$,

where $x=V / V_{0}$ is the relative volume change, $V_{0}$ is the unit cell volume at $P=0, B_{0}=-V(d P / d V)_{T}$ is the bulk

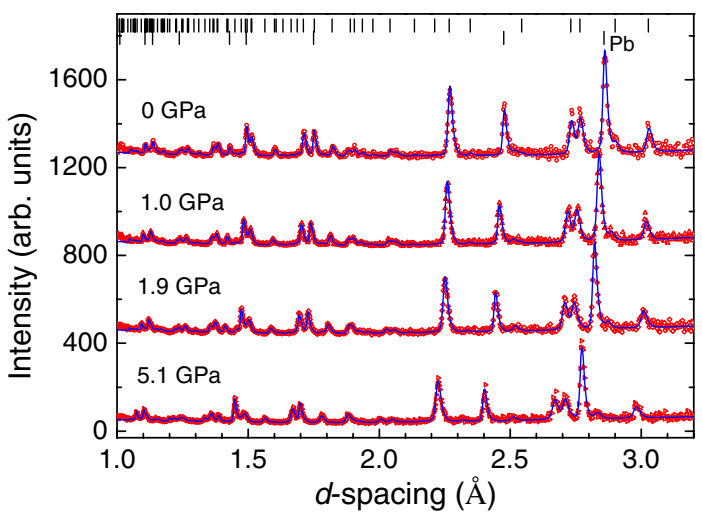

FIG. 1 (color online). Neutron diffraction patterns of deuterated herbertsmithite measured at selected pressures and ambient temperature and refined by the Rietveld method. Experimental points and calculated profiles (solid line) are shown. The calculated positions of diffraction peaks for herbertsmithite (upper row) and $\mathrm{Pb}$ (lower row) used as a pressure marker are shown at the top.

modulus and, $B^{\prime}=\left(d B_{0} / d P\right)_{T}$ is the pressure derivative of the bulk modulus. The value $B_{0}=70(4) \mathrm{GPa}$ was obtained with the fixed $B^{\prime}=4$ and the experimental value of $V_{0}=$ $570.9(3) \AA^{3}$.

The $\mathrm{Cu}-\mathrm{O}$ bond distance and $\mathrm{Cu}-\mathrm{O}-\mathrm{Cu}$ bond angle exhibit anomalous behavior upon compression at ambient temperature. In the pressure range $0-2.5 \mathrm{GPa}$ the $\mathrm{Cu}-\mathrm{O}$ bond distance decreases linearly with the pressure coefficient $\quad k_{\mathrm{Cu}-\mathrm{O}}=-\left(1 /\left(l_{\mathrm{Cu}-\mathrm{O}}\right)_{P=0}\right)\left(d l_{\mathrm{Cu}-\mathrm{O}} / d P\right)_{T}=$ $0.0075 \mathrm{GPa}^{-1}$, while it becomes nearly pressure independent for $P>2.5 \mathrm{GPa}$ (Fig. 2). In contrast, the $\mathrm{Cu}-\mathrm{Cl}$ distance exhibits a linear decrease upon compression with a pressure coefficient $k_{\mathrm{Cu}-\mathrm{Cl}}=0.0069 \mathrm{GPa}^{-1}$. The $\mathrm{Cu}-\mathrm{O}-\mathrm{Cu}$ bond angle mediating the strong superexchange AFM interaction between neighboring $\mathrm{Cu}^{2+}$ ions within the kagome layers shows a small linear increase from 118.1 (4) to $119.1(4)^{\circ}$ in the $0-2.5 \mathrm{GPa}$ pressure range, which is followed by a decrease to $116.4(4)^{\circ}$ upon further compression at $5.1 \mathrm{GPa}$ (Fig. 2). This increased bending is accompanied by a tilting of the $\mathrm{CuO}_{4}$ planes away from the $(a b)$ plane of the crystal structure containing the kagome layer (Fig. 3). The $\mathrm{Cu}-\mathrm{Cl}-\mathrm{Cu}$ bond angle, on the other hand, exhibits only a slight increase from $76.0(2)$ to $76.4(2)^{\circ}$ in the $0-2.5 \mathrm{GPa}$ pressure range and remains nearly unchanged at higher pressures up to $5.1 \mathrm{GPa}$ (Fig. 2). As a result, the $\mathrm{CuO}_{4}$ plane tilts with respect to the $\mathrm{Cl}-\mathrm{Cu}-\mathrm{Cl}$ axis of each $\mathrm{CuO}_{4} \mathrm{Cl}_{2}$ octahedron (Fig. 3), so that above $2.5 \mathrm{GPa}$ the sixfold coordination of the $\mathrm{Cu}^{2+}$ cations comes slightly closer to $D_{4 h}$ (trigonally distorted octahedral), with the larger $\mathrm{O}-\mathrm{Cu}-\mathrm{Cl}$ angles decreasing from 97.1(4) to 96.7 $(4)^{\circ}$. No such changes are observed in the $D_{3 d}$ crystal field of the $\mathrm{Zn}$ cations. Remarkably, despite the differences in the response of the individual bonds and angles to applied pressure, the pressure dependence of the unit cell volume remains practically linear up to $5.1 \mathrm{GPa}$. While this is 

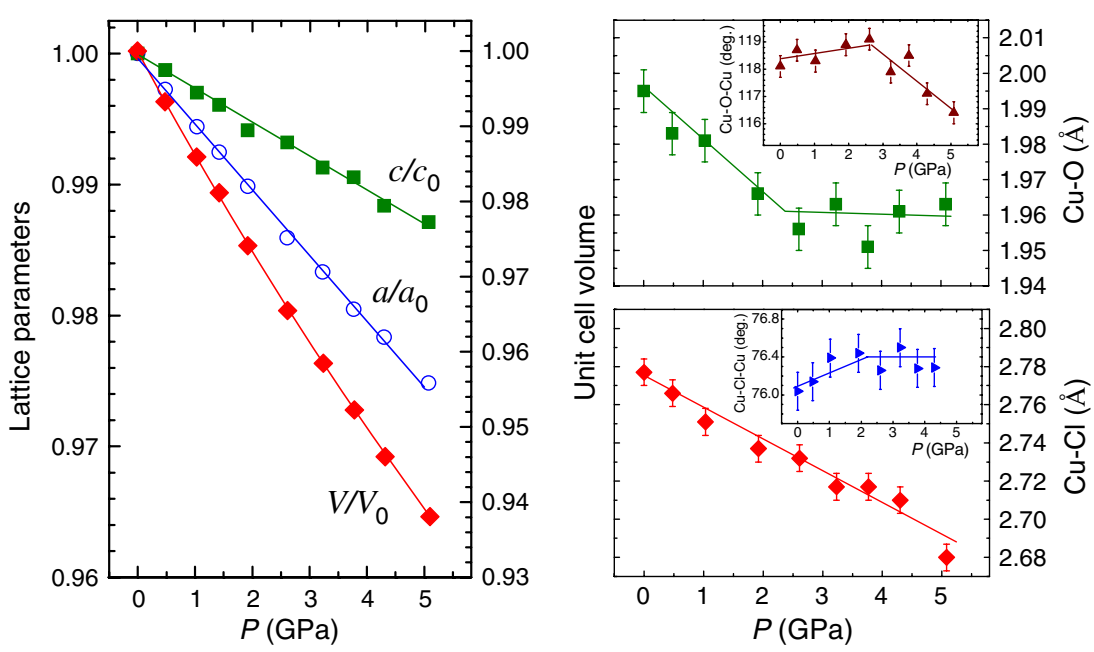

FIG. 2 (color online). Left: Lattice parameters and unit cell volume of deuterated herbertsmithite as functions of pressure interpolated by linear functions and Birch-Murnaghan equation of state, respectively. Right: Interatomic distances $\mathrm{Cu}-\mathrm{O}, \mathrm{Cu}-\mathrm{Cl}$ and bond angles $\mathrm{Cu}-\mathrm{O}-\mathrm{Cu}, \mathrm{Cu}-\mathrm{Cl}-\mathrm{Cu}$ (insets) as functions of pressure. The lines are guides to the eye only.

surprising, it should be noted that the anomalies observed in the behavior of some structural parameters are not discontinuities but rather abrupt changes in their pressure dependence, arising from the complex interplay between the compression of interatomic bonds and the tilting of $\mathrm{CuO}_{4} \mathrm{Cl}_{2}$ octahedral units.

The magnetic pressure-temperature phase diagram based on the magnetic susceptibility measurements is shown in Fig. 4(a). The appearance of a peak associated with the onset of the long-range antiferromagnetic order is observed at $P=2.5 \mathrm{GPa}$ and $T_{N}=6 \mathrm{~K}$. Upon further pressure increase the magnetic ordering temperature decreases slightly to $4.5 \mathrm{~K}$ at $P=10 \mathrm{GPa}$. The pressure of $2.5 \mathrm{GPa}$, at which the transition from the quantumdisordered state to the classical Néel-ordered antiferromagnetic state is observed, correlates with the observed anomaly in the pressure dependence of the $\mathrm{Cu}-\mathrm{O}$ distance and the $\mathrm{Cu}-\mathrm{O}-\mathrm{Cu}$ angle at room temperature. The electrical resistivity measurements confirmed herbertsmithite remains insulating over the entire pressure range.

A low-temperature high-pressure neutron diffraction experiment was carried out to look for magnetic Bragg peaks in the potentially spin-frozen phase observed below $\sim 6 \mathrm{~K}$ in the magnetic susceptibility measurements above $2.5 \mathrm{GPa}$. For the analysis of the magnetic contribution to the diffraction spectra, the difference pattern obtained by subtraction of the $50 \mathrm{~K}$ data (above $T_{N}$ ) from the $1.4 \mathrm{~K}$ data was used [Fig. 4(b)]. By following this method, two weak magnetic reflections were identified in the $1.4 \mathrm{~K}$ data, at $Q \approx 1.33$ and at $1.26 \AA^{-1}$.

There are two types of long-range magnetic order which are commonly considered in kagome antiferromagnets. These are the so-called " $q=0$ " structure with a positive vector chirality and the " $\sqrt{3} \times \sqrt{3}$ " structure with a staggered vector chirality [25]. From the Rietveld refinement it was found that the positions and the intensities of the observed magnetic reflections are consistent with the $\sqrt{3} \times \sqrt{3}$ magnetic structure [Fig. 4(b)] of herbertsmithite, while the $q=0$ model is in poor agreement with the data. The estimated ordered moment at $T=1.4 \mathrm{~K}$ is refined as $\mu=0.8(1) \mu_{B}$, which is comparable to the ordered moment of $\mu=0.6 \mu_{B}$ found in the related compound clinoatacamite, $\mathrm{Cu}_{4}(\mathrm{OD})_{6} \mathrm{Cl}_{2}$ [26]. We acknowledge that although the presence of the magnetic peaks and the nature of the magnetic order have been established, due to the complexity of the high-pressure neutron powderdiffraction experiments at very low temperatures,

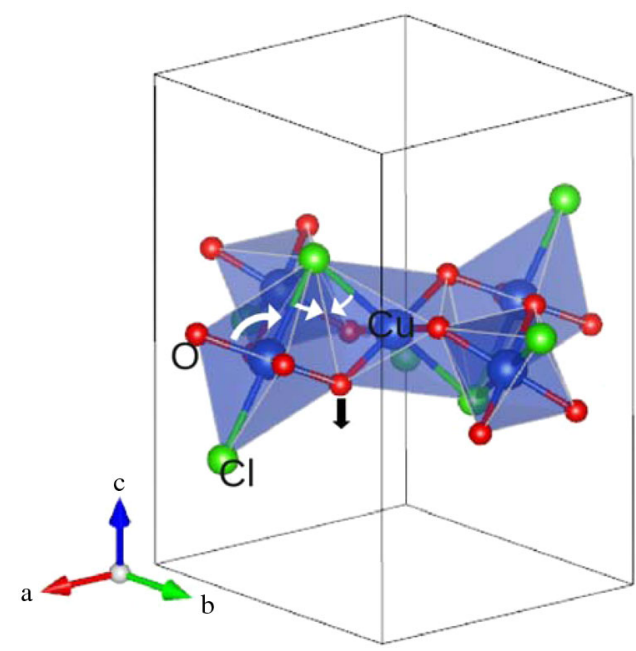

FIG. 3 (color online). An illustration of the structural response of the deuterated herbertsmithite under high pressure. The tilting of the $\mathrm{CuO}_{4}$ plane with respect to the $\mathrm{Cl}-\mathrm{Cu}-\mathrm{Cl}$ axis and modification of $\mathrm{O}-\mathrm{Cu}-\mathrm{Cl}$ and $\mathrm{Cu}-\mathrm{O}-\mathrm{Cu}$ bond angles, leading to the trigonal distortion of the octahedral coordination, are indicated by arrows. 


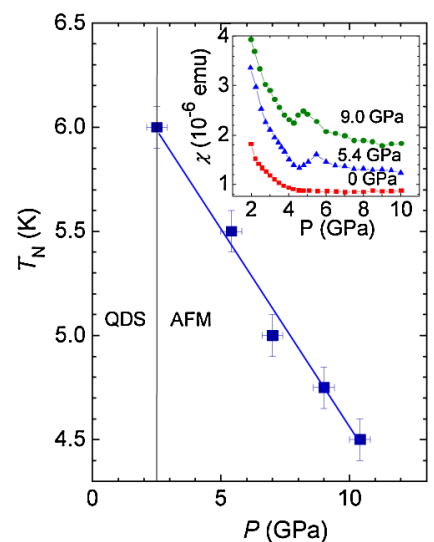

(a)

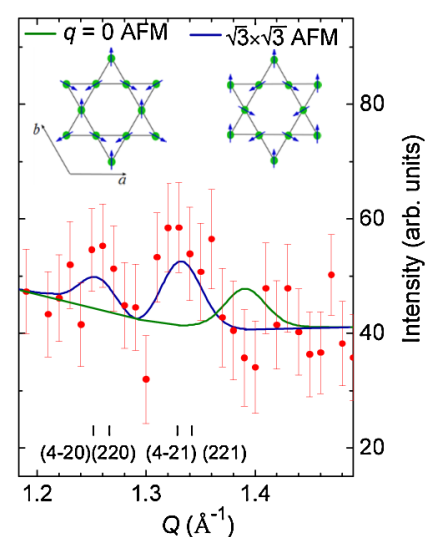

(b)
FIG. 4 (color online). (a) Magnetic pressure-temperature phase diagram of herbertsmithite, the regions of the quantumdisordered (QDS) and the classical Néel (AFM) states are shown. The vertical line represents the phase boundary between these states. The inset represents magnetic susceptibility data collected at selected pressures. (b) Difference pattern of herbertsmithite, obtained by subtraction of $50 \mathrm{~K}$ neutron diffraction data from $1.4 \mathrm{~K}$ data, both measured at $2.7 \mathrm{GPa}$. The calculated profiles for the $\sqrt{3} \times \sqrt{3}$ and $q=0$ AFM models are shown. The indices of the observed magnetic peaks are given below (for the $\sqrt{3} \times \sqrt{3}$ magnetic cell).

conducted on small samples with the volume of about $1 \mathrm{~mm}^{3}$, and the weak magnetic moment of the $\mathrm{Cu}^{2+}$ ions, further experiments using single-crystal neutron diffraction would be helpful to fully characterize the AFM ordered state in the high-pressure phase of herbertsmithite.

It is well established that there is a magnetic anisotropy in herbertsmithite, that has been attributed to a Dzyaloshinsky-Moriya interaction (DMI) [10,12,27]. Anisotropies, of the DMI-type or otherwise, can also induce a long-range order in kagome antiferromagnets as is well documented for the jarosites with $S=5 / 2$ [28]. For the low-spin case the DM interaction is the most likely cause of the anisotropy in kagome-lattice materials, where the Hamiltonian takes the form

$$
H=\sum_{n n}\left[J \hat{S}_{i} \cdot \hat{S}_{j}+\vec{D}_{i j} \cdot\left(\hat{S}_{i} \times \hat{S}_{j}\right)\right],
$$

where $J$ is the superexchange interaction strength and $\vec{D}_{i j}= \pm \hat{e}_{z} D_{z}$ is the Dzyaloshinsky-Moriya anisotropy vector taken perpendicular to the kagome planes and staggered between neighboring kagome triangles. This Hamiltonian has been studied using finite-size scaling of exact diagonalization results of clusters [12] and recently also using Schwinger-boson mean-field theory [29]. The exact diagonalization calculations pointed to a secondorder transition to the Néel state on increasing of $D_{z}$ from the ambient pressure value of $0.08 J[10]$ to the critical value $\sim 0.1 J$. The Schwinger-boson mean-field theory, in which the spin quantum number $S$ becomes a variable (bosonic density), resembles the exact diagonalization results for a small Schwinger-boson density $S \sim 0.2$, while it predicts the $\sqrt{3} \times \sqrt{3}$ ground state for $S=0.5$ and a small $D_{z} / J<0.04$. The change in the ratio of the Dzyaloshinsky-Moriya coupling to the superexchange coupling $D_{z} / J$ is proportional to $\lambda / \Delta_{\text {cf }}$, where $\lambda$ is the spin-orbit coupling constant and $\Delta_{\text {cf }}$ is the crystal-field splitting [30]. The spin-orbit coupling constant is related to intra-atomic interactions and it is weakly pressure dependent $[31,32]$. The variation of the crystal-field splitting upon compression can be evaluated as $\Delta_{\mathrm{cf}} \sim\left(l_{\mathrm{Cu}-\mathrm{O}}\right)^{-5}$, where $l_{\mathrm{Cu}-\mathrm{O}}$ is the $\mathrm{Cu}-\mathrm{O}$ bond length [31]. One can estimate a $9 \%$ reduction in the quantity $D_{z} / J$ due to the application of pressure of $2.5 \mathrm{GPa}$.

Therefore, the observed pressure-induced onset of the $\sqrt{3} \times \sqrt{3}$ magnetic order on decrease of $D_{z} / J$ is not consistent with the phase diagram predicted by the exact diagonalization. However, it can be explained in terms of the phase diagram obtained by the Schwinger-boson meanfield theory for relatively small $D_{z} / J$, if one assumes an increase of the bosonic density $S$ with pressure. One could speculate whether an effective increase in the spin quantum number with pressure could arise due to the enhanced spinorbit coupling as the electron density and orbital momentum increase upon compression. It is also worth noting that in the absence of the Dzyaloshinsky-Moriya coupling the $\sqrt{3} \times \sqrt{3}$ ground state is selected for the kagome-lattice Heisenberg antiferromagnet in the case of relatively large $S$ values by quantum fluctuations ("order by disorder" mechanism) [33]. The nature of the spin-freezing transition in herbertsmithite is different from those observed under pressure in other spin-liquids such as $\mathrm{Tb}_{2} \mathrm{Ti}_{2} \mathrm{O}_{7}$, mediated by the uniaxial stress component [14], or $\mathrm{TlCuCl}_{3}$ and $\mathrm{KCuCl}_{3}$, mediated by the closing of the spin-energy gap $[15,16]$. Further theoretical studies taking into account the interplay between Dzyaloshinsky-Moriya coupling and the quantum fluctuations would be important to clarify the balance between these factors in the formation of the phase diagram of the herbertsmithite. However, other explanations for the onset of the long-range order, such as those related to the increased further-neighbor interactions, cannot at this stage be ruled out.

In the antiferromagnetically ordered state the value of the Néel temperature is controlled by the strength of the superexchange interaction $J \sim \cos ^{2}\left(\theta_{\mathrm{Cu}-\mathrm{O}-\mathrm{Cu}}\right) /\left(l_{\mathrm{Cu}-\mathrm{O}}\right)^{\mathrm{n}}$, $n \sim 10$ [34]. At pressures above $2.5 \mathrm{GPa}$ the $\mathrm{Cu}-\mathrm{O}$ bond length remains almost constant, while the $\mathrm{Cu}-\mathrm{O}-\mathrm{Cu}$ bond angle decreases. Such structural behavior should result in the reduction of the $J$ value by $15 \%$ between 2.5 and $5.1 \mathrm{GPa}$, explaining the observed decrease in $T_{N}$. Figure 4(a) suggests the spin-freezing transition is of the first order, but this could be due to the insufficiently small steps in pressure at which the data were taken in the vicinity of $2.5 \mathrm{GPa}$. If with pressure we reach the same ordered phase as accessed with applied magnetic fields [13] then this should be a second-order transition. 
The results of our study demonstrate that herbertsmithite exhibits a phase transition from the quantum-disordered spin liquid to the long-range ordered antiferromagnetic state upon compression. The $\sqrt{3} \times \sqrt{3}$ symmetry unit cell of the AFM order in the high-pressure phase is compatible with the neutron diffraction data. The observed magnetic phase diagram of the herbertsmithite points towards a complex interplay of Dzyaloshinskii-Moriya coupling, quantum fluctuations, and possibly further-neighbor interactions, requiring further theoretical clarification.

We would like to thank Stephen Moggach for providing single-crystal x-ray diffraction data and Karl Syassen, Elissaios Stavrou, and Ingo Loa for useful discussions. We gratefully acknowledge the technical assistance of Robert Lauden and Paul Aitken of CSEC. This work was funded by and supported through resources made available by the Science and Technology Facilities Council and the Russian Foundation for Basic Research, Grant No. 12-0200794-a.

[1] G. Misguich and C. Lhuillier, Frustrated Spin Systems, edited by H. T. Diep (World Scientific, Singapore, 2005).

[2] P. W. Anderson, Science 235, 1196 (1987)

[3] M.P. Shores, E. A. Nytko, B. M. Bartlett, and D. G. Nocera, J. Am. Chem. Soc. 127, 13462 (2005).

[4] P. Mendels, F. Bert, M. A. de Vries, A. Olariu, A. Harrison, F. Duc, J. C. Trombe, J. S. Lord, A. Amato, and C. Baines, Phys. Rev. Lett. 98, 077204 (2007).

[5] J. S. Helton et al., Phys. Rev. Lett. 98, 107204 (2007).

[6] G. Misguich and P. Sindzingre, Eur. Phys. J. B 59, 305 (2007).

[7] M. A. de Vries, J.R. Stewart, P. P. Deen, J. O. Piatek, G. J. Nilsen, H. M. Rønnow, and A. Harrison, Phys. Rev. Lett. 103, 237201 (2009).

[8] A. Olariu, P. Mendels, F. Bert, F. Duc, J. C. Trombe, M. A. de Vries, and A. Harrison, Phys. Rev. Lett. 100, 087202 (2008).

[9] T. Imai, E. A. Nytko, B. M. Bartlett, M. P. Shores, and D. G. Nocera, Phys. Rev. Lett. 100, 077203 (2008).

[10] A. Zorko, S. Nellutla, J. van Tol, L. C. Brunel, F. Bert, F. Duc, J.-C. Trombe, M. A. de Vries, A. Harrison, and P. Mendels, Phys. Rev. Lett. 101026405 (2008).

[11] M. A. de Vries, K. V. Kamenev, W. A. Kockelmann, J. Sanchez-Benitez, and A. Harrison, Phys. Rev. Lett. 100, 157205 (2008).
[12] O. Cepas, C. M. Fong, P. W. Leung, and C. Lhuillier, Phys. Rev. B 78, 140405(R) (2008).

[13] M. Jeong, F. Bert, P. Mendels, F. Duc, J. C. Trombe, M. A. de Vries, and A. Harrison, Phys. Rev. Lett. 107, 237201 (2011).

[14] I. Mirebeau, I. N. Goncharenko, G. Dhalenne, and A. Revcolevschi, Phys. Rev. Lett. 93, 187204 (2004).

[15] Ch. Ruegg, A. Furrer, D. Sheptyakov, Th. Strässle, K. W. Krämer, H.-U. Güdel, and L. Mélési, Phys. Rev. Lett. 93, 257201 (2004).

[16] K. Goto, M. Fujisawa, H. Tanaka, Y. Uwatoko, A. Oosawa, T. Osakabe, and K. Kakurai, J. Phys. Soc. Jpn. 75, 064703 (2006).

[17] J. M. Besson, R. J. Nelmes, G. Hamel, J. S. Loveday, G. Weill, and S. Hull, Physica (Amsterdam) 180-181B , 907 (1992).

[18] W. G. Marshall and D. J. Francis, J. Appl. Crystallogr. 35, 122 (2002)

[19] O. Schulte and W. B. Holzapfel, Phys. Rev. B 52, 12636 (1995).

[20] V.P. Glazkov and I. N. Goncharenko, Fizika I Tekhnika Vysokih Davlenij 1, 56 (1991).

[21] J. Rodríguez-Carvajal, Physica (Amsterdam) 192B, 55 (1993).

[22] G. Giriat, W. Wang, J. Paul Attfield, A. D. Huxley, and K. V. Kamenev, Rev. Sci. Instrum. 81, 073905 (2010).

[23] K. V. Kamenev, J. Sanchez-Benitez, and S. Tancharakorn, High Press. Res. 27, 189 (2007).

[24] F. J. Birch, J. Geophys. Res. 91, 4949 (1986).

[25] D. Grohol, K. Matan, J.-H. Cho, S.-H. Lee, J. W. Lynn, D. G. Nocera, and Y.S. Lee, Nature Mater. 4, 323 (2005).

[26] A. S. Wills and J.-Y. Henry, J. Phys. Condens. Matter 20, 472206 (2008).

[27] O. Ofer and A. Keren, Phys. Rev. B 79, 134424 (2009)

[28] M. De Vries, T. K. Johal, A. Mirone, J. S. Claydon, G. J. Nilsen, H. M. Rønnow, G. van der Laan, and A. Harrison, Phys. Rev. B 79, 045102 (2009).

[29] L. Messio, O. Cepas, and C. Lhuillier, Phys. Rev. B 81, 064428 (2010).

[30] M. Elhajal, B. Canals, and C. Lacroix, Phys. Rev. B 66, 014422 (2002).

[31] A. Abragam and B. Bleaney, Electron Paramagnetic Resonance of Transition Ions (Clarendon, Oxford, 1970), p. 21 and p.443.

[32] E. Francisco and L. Pueyo, Phys. Rev. B 37, 5278 (1988).

[33] S. Sachdev, Phys. Rev. B 45, 12377 (1992).

[34] J. B. Goodenough, Rep. Prog. Phys. 67, 1915 (2004). 